\title{
Fracturas por avulsión bilateral de tuberosidades tibiales en un adolescente, reinserción y protección con banda de tensión: reporte de un caso
}

\author{
Bilateral avulsion fractures of the tibial tuberosity in an adolescent, \\ reinsertion and protection with a tension band: a case report
}

\author{
Gonzalo Rafael Balderas Rodríguez, * Mónica del Socorro Ortiz Mesina, ${ }^{\ddagger}$ José Armando Rodríguez Hernández, ${ }^{\S}$ \\ Jorge Alberto Contreras Córdoba, $\$$ Carlos Alberto Ochoa Contreras $\$$ \\ *Médico Residente de Traumatología y Ortopedia. Hospital General de Zona No. 1; ‡Doctora en Ciencias. \\ Coordinación de Planeación y Enlace Institucional; §Traumatólogo Ortopedista. Hospital General de Zona No. 1. \\ Instituto Mexicano del Seguro Social, Colima.
}

\begin{abstract}
Resumen
Las fracturas por avulsión bilateral de tuberosidades tibiales son un padecimiento muy raro, con una incidencia que varía de 0.4 a $2.7 \%$ del total de las lesiones epifisiarias. Los manejos más frecuentes reportados en la literatura son reducción abierta y fijación interna con tornillos más inmovilización con aparatos de yeso por cuatro a seis semanas. Presentamos el caso de un paciente masculino de 14 años de edad con antecedente de enfermedad de Osgood-Schlatter que sufre fracturas, avulsión bilateral de tuberosidades tibiales tipo Ogden IIA secundario a contracción enérgica del cuádriceps al evitar caída de patineta. Describimos un tratamiento exitoso con reducción abierta y fijación interna con tornillos de $4.0 \mathrm{~mm}$, arandelas y protección con bandas de tensión, sin la necesidad de inmovilización con aparatos ortopédicos. Movilización inmediata al segundo día postquirúrgico. Alos tres meses de evolución, se retira material de osteosíntesis por fatiga de bandas de tensión, encontrándose tuberosidades ya con datos de consolidación y movilidad completa, sin interrumpir la movilización activa. Al año de evolución se encuentra con rodillas sin deformidades, indoloras y practicando actividad deportiva, sin requerir de rehabilitación física exhaustiva o la incomodidad de cualquier tipo de inmovilización. Resulta efectivo el manejo con tornillos de compresión y bandas de tensión en adolescentes con este padecimiento y eliminamos las secuelas que conlleva la inmovilización en un paciente postoperado.
\end{abstract}

Palabras clave: Fracturas de tibia, reducción de fracturas abiertas, lesiones de rodilla, fracturas, avulsión.

\section{Abstract}

Bilateral avulsion fractures of the tibial tuberosity are a very rare condition, with an occurrence rate ranging from 0.4 to $2.7 \%$ of all epiphyseal injuries. The most frequent approaches reported in the literature are open reduction and internal fixation with screws plus cast immobilization for four to six weeks. We present the case of a 14-year-old male patient with a history of Osgood-Schlatter disease suffering from bilateral avulsion fractures of the tibial tuberosity type Ogden IIA secondary to energetic contraction of the quadriceps to avoid falling from a skateboard. We describe a successful treatment with open reduction and internal fixation with $4.0 \mathrm{~mm}$ screws, washers, and protection with tension bands without the need of immobilization with orthopedic devices. Immediate mobilization on the second day post-surgical. After three months of evolution, the tension band fatigue osteosynthesis material is removed, finding tuberosities with consolidation data and complete mobility, without interrupting active mobilization. After a year of evolution, we found knees without deformities, painless and practicing sports activity, without requiring thorough physical rehabilitation or the discomfort caused by any type of immobilization. The treatment with compression screws and tension bands on teenagers with this condition proves to be effective, moreover, we eliminated the aftermath derived from the immobilization of a patient, post-surgery.

Keywords: Tibial fracture, reduction of open fractures, knee injuries, fractures, avulsion.

Correspondencia:

Carlos Alberto Ochoa Contreras

E-mail: carlos8acontreras@gmail.com

Recibido: 24-05-2021. Aceptado: 15-06-2021.
Citar como: Balderas RGR, Ortiz MMS, Rodríguez HJA, Contreras CJA, Ochoa CCA. Fracturas por avulsión bilateral de tuberosidades tibiales en un adolescente, reinserción y protección con banda de tensión: reporte de un caso. Orthotips. 2021;17 (4):237-241. https:// dx.doi.org/10.35366/102222 


\section{Introducción}

Las fracturas por avulsión de tuberosidades tibiales tienen una incidencia de 0.4 a $2.7 \%$ de las lesiones epifisarias. ${ }^{1}$ Hasta el momento, sólo se han reportado 28 casos desde 1955, Borsch-Madsen fue el primero en describirlo. ${ }^{1-18}$ Ocurre en el centro de osificación anterior de la tibia, que osifica entre los 13 y 15 años en niñas y entre los 15 y 19 años en niños. ${ }^{2,19}$ Existen dos mecanismos de lesión: contracción enérgica del cuádriceps con la rodilla en extensión o flexión forzada de la rodilla. ${ }^{12}$ Se ha asociado con Osgood-Schlatter ${ }^{20,21}$ o deficiencia de vitamina $D,{ }^{4}$ aunque también podría estar relacionado con trastornos del tejido conectivo. ${ }^{12}$

Watson-Jones en 1976 fue el primero en clasificarlo en tipo I: fractura de la tuberosidad tibial, sin afección de la epífisis proximal; tipo II: con afección de la epífisis proximal; y tipo III: con extensión a la superficie articular de la rodilla. En 1980 Ogden la subdivide según el desplazamiento de la fractura, en tipo A sin desplazamiento y tipo B muy desplazada. Ryu en 1985 agrega un tipo IV con involucro posterior de la epífisis proximal y en 2003 McKoy agrega un tipo $V$ con un patrón de fractura en « $Y » .13$

El tratamiento ortopédico recomendado es aparato de yeso por cuatro a seis semanas en el tipo I de Watson-Jones y reducción abierta y fijación interna a partir del tipo II, ${ }^{3,12}$ ya sea con alambres de Kirschner, bandas de tensión, cerclajes o tornillos canulados más colocación de aparato de yeso por tres a seis sema-
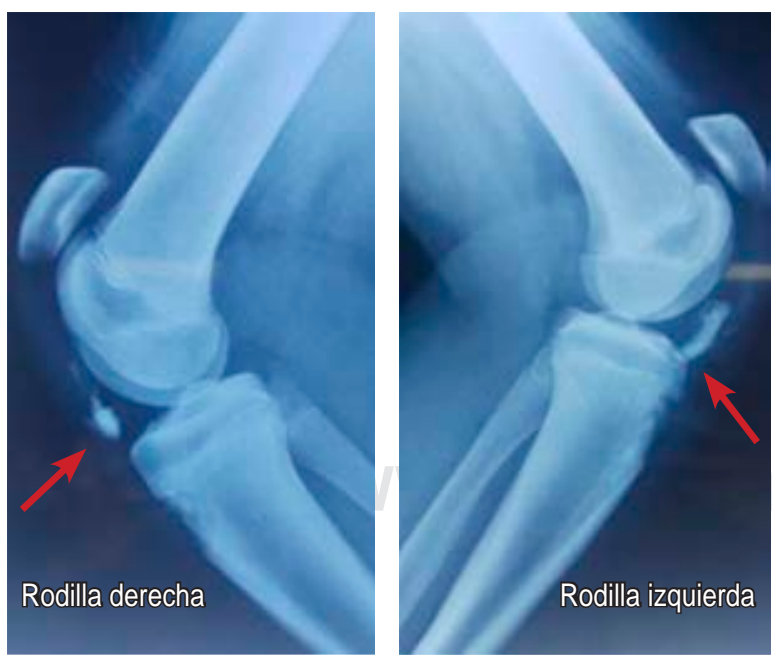

Figura 1: Radiografía lateral de ambas rodillas, con fracturas por avulsión de tuberosidades tibiales (flechas rojas), clasificación Ogden IIA para ambas rodillas.

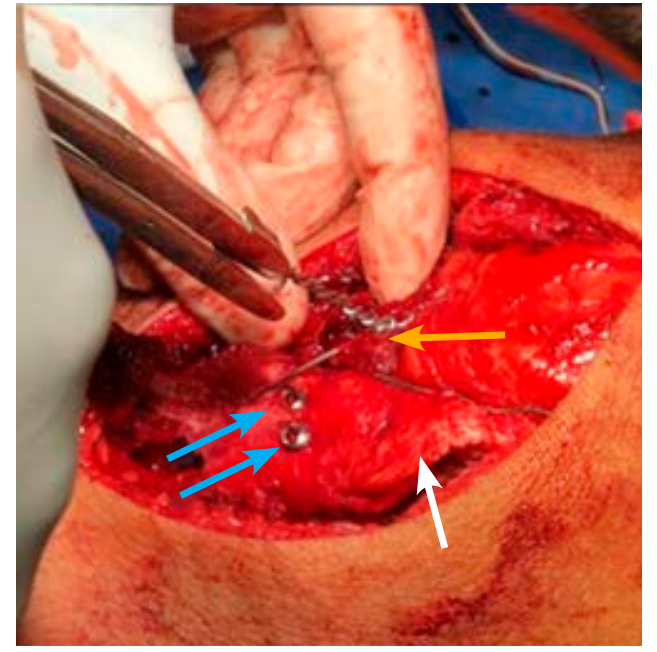

Figura 2: Foto transquirúrgica de rodilla izquierda donde se observa tendón rotuliano íntegro (flecha blanca), reducción abierta y fijación interna a tuberosidades tibiales con dos tornillos de esponjosa de 4.0 $\mathrm{mm}$ realizando compresión (flechas azules) y bandas de tensión para protección (flecha amarilla).

nas, soportar peso con ayuda de bastón o muletas a las seis semanas y deambulación independiente a los tres a seis meses. ${ }^{1-18}$

Biomecánicamente el tendón del cuádriceps tracciona la fractura de la tuberosidad tibial, las bandas de tensión pueden neutralizar estas fuerzas. Nikiforidis y colaboradores describen el procedimiento con alambre quirúrgico abrazando el polo superior de la rótula y después fijándolo a la tibia, por debajo de la tuberosidad tibial, ${ }^{18}$ así evitando el desplazamiento de la fractura al momento de la movilización temprana y con ello disminuyendo el riesgo de artrofibrosis y pérdida de masa muscular. ${ }^{5}$

Algunas de las complicaciones descritas son: irritación de la piel por el material de osteosíntesis, ${ }^{16}$ embolia pulmonar, ${ }^{17}$ epifisiodesis temprana ${ }^{7}$ y síndrome compartimental. ${ }^{17}$

\section{Presentación del caso}

Masculino de 14 años, con antecedente de Osgood-Schlatter diagnosticado a los siete años, que inicia padecimiento al evitar caer de patineta, seguido de dolor intenso en ambas rodillas e imposibilidad a la bipedestación. A la exploración física con edema en rodillas, con actitud en flexión de $30^{\circ}$, imposibilidad a la extensión activa. A la palpación rótula alta y tuberosidad tibial desplazada en ambas rodillas. 
Evaluación radiográfica con fractura de tuberosidad tibial Ogden IIA, para ambas rodillas (Figura 1). Se descarta por análisis laboratorial deficiencia de vitamina $\mathrm{D}$ o alguna otra alteración endocrinológica. Se aplican criterios de Beighton-Horan descartando síndrome de hipermovilidad o alguna otra afección de la colágena.

Se realiza intervención quirúrgica, encontrándose lesión de retináculo medial bilateral, tendón rotuliano íntegro, tuberosidades tibiales se fijan con dos tornillos de esponjosa 4.0, arandelas y bandas de tensión con alambre $1.2 \mathrm{~mm}$ a $45^{\circ}$ de flexión (Figura 2). Movilización activa a $60^{\circ}$ de flexión y $0^{\circ}$ de extensión de rodillas en postquirúrgico inmediato. No es posible retirar bandas de tensión a la sexta semana, aun así el paciente ya contaba con arcos de movilidad completos y deambulación sin ayuda de muletas. Tres meses y medio postquirúrgico inicia con dolor repentino e imposibilidad a la deambulación, estudio radiográfico muestra fatiga de bandas de tensión y tuberosidades tibiales con datos de consolidación (Figura 3), por lo que se retira material de osteosíntesis. Al año de evolución se encuentran fracturas consolidadas (Figura 4), sin deformidad clínica en rodillas (Figura 5), sin dolor residual y realizando actividades deportivas.

\section{Discusión}

Las fracturas con avulsión de tuberosidad tibial bilateral son extremadamente raras. ${ }^{16}$ Se han asocia-
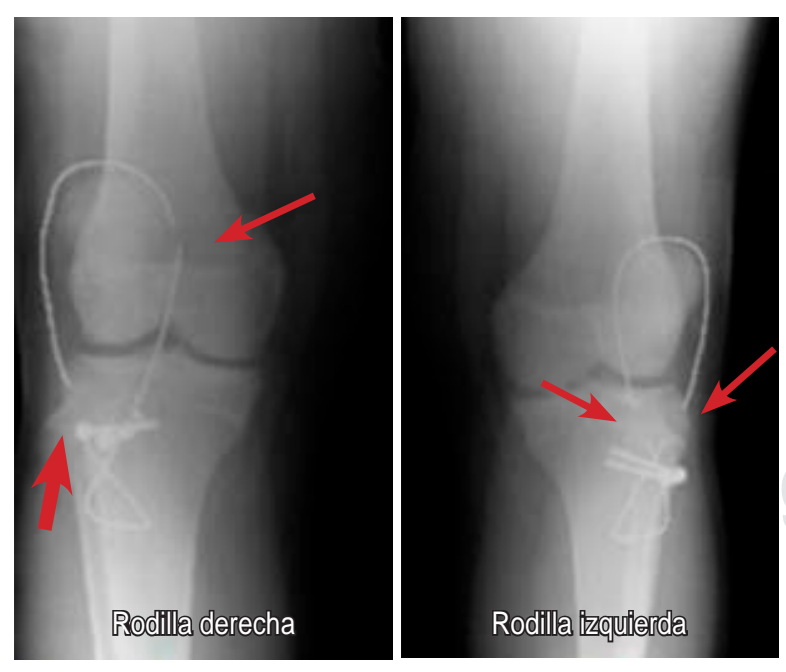

Figura 3: Radiografía anteroposterior de ambas rodillas, tres meses y medio después de evento quirúrgico, muestra fatiga de alambre 1.2 $\mathrm{mm}$ (flechas rojas).
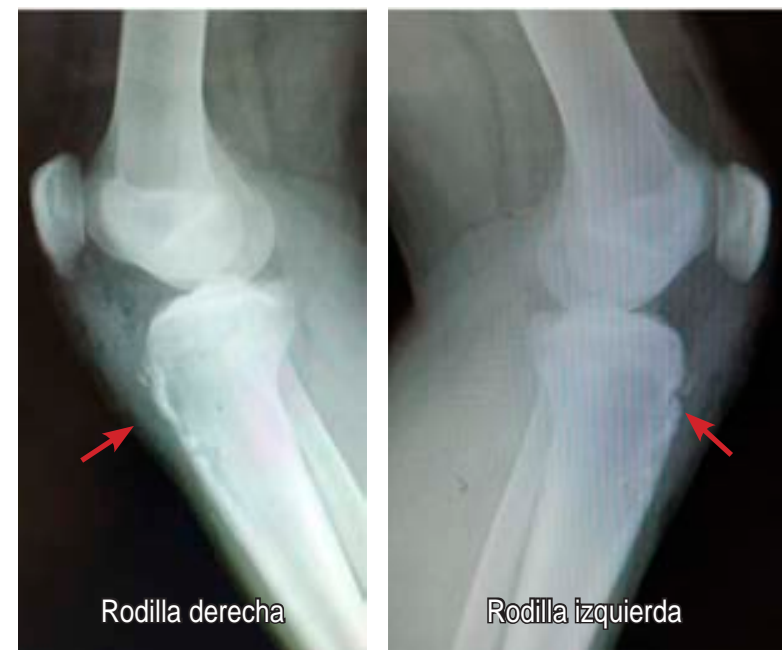

Figura 4: Radiografía lateral de ambas rodillas, un año después del evento quirúrgico, muestra fracturas por avulsión de tuberosidades tibiales ya consolidadas (flecha roja).
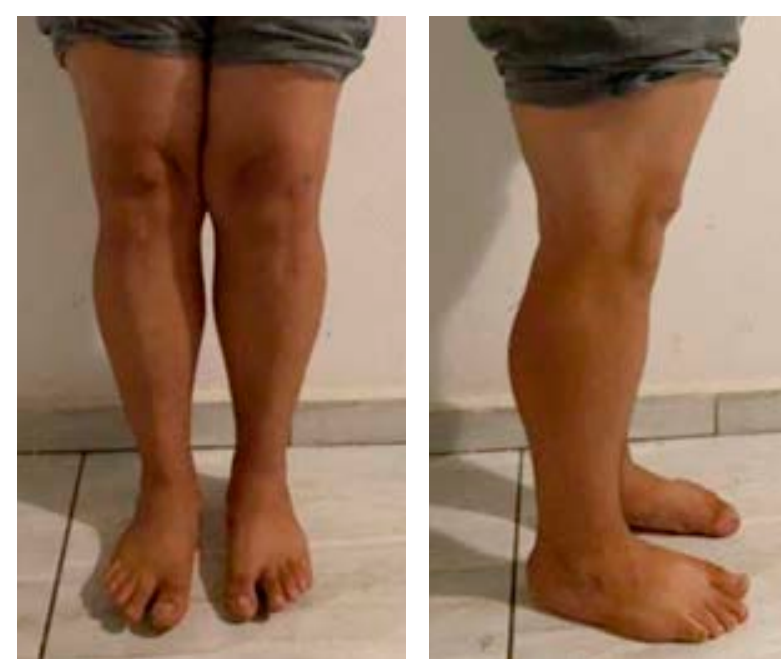

Figura 5: Fotografías de paciente en bipedestación al año de evolución. A) Sin deformidad clínica. B) Sin recurvatum o antecurvatum en rodillas y miembros pélvicos normotróficos.

do con enfermedad de Osgood-Schlatter ${ }^{17,20,21}$ y en este caso tenía el antecedente desde los siete años, lo que podría favorecer la fractura; asimismo, también contribuye la debilidad del centro de osificación secundario de la tibia a causa del cambio de tejido fibrocartilaginoso por células columnares. ${ }^{19}$ También se descarta deficiencia de vitamina $D,{ }^{4}$ osteogénesis imperfecta ${ }^{17}$ e hipermovilidad articular benigna, y al no contar con antecedentes heredofamiliares y no 
cumplir con criterios de Beighton-Horan, se descarta cualquier alteración de la colágena.22,23

Los tornillos canulados con colocación de aparato de yeso por tres a seis semanas son el tratamiento más utilizado. Sin embargo, Ergün y colaboradores obtuvieron limitación de los arcos de movilidad, ${ }^{6}$ Slobogean y su equipo describen deformidad de rodilla con $5^{\circ}$ de flexión, ${ }^{11}$ Newman y su grupo disminución de la masa muscular del cuádriceps con limitación de los arcos de movilidad, ${ }^{5}$ por lo que en este caso decidimos colocar tornillos de esponjosa con uso de bandas de tensión como protección y movilización activa en el postquirúrgico inmediato, ya que la inmovilidad de la rodilla provoca aumento del factor de crecimiento fibroblástico B1 que da proliferación patológica de fibroblastos, formando tejido conectivo en exceso y liberación de citocinas, con mayor inflamación. ${ }^{24}$ Obtuvimos buenos resultados en la consolidación y en la movilidad a los 12 meses postquirúrgicos, sin necesitad de rehabilitación física exhaustiva y sin la incomodidad de cualquier tipo de inmovilización.

\section{Conclusiones}

El tratamiento óptimo de las fracturas por avulsión bilateral de la tuberosidad tibial es la reducción abierta y la fijación interna para procurar la movilización temprana; en este caso, al ser fractura por avulsión bilateral de tuberosidades tibiales grado IIA de Ogden, resultó efectivo el manejo con tornillos de compresión y protección con bandas de tensión, para realizar una movilización inmediata que disminuyó el riesgo de artrofibrosis sin la pérdida de masa muscular significativa, por lo que no requirió de una terapia física exhaustiva, a su vez se evitó la incomodidad de cualquier tipo de inmovilización. Con un resultado óptimo a los tres meses de evolución.

\section{Referencias}

1. Georgiou G, Dimitrakopoulou A, Siapkara A, Kazakos K, Provelengios S, Dounis E. Simultaneous bilateral tibial tubercle avulsion fracture in an adolescent: a case report and review of the literature. Knee Surg Sports Traumatol Arthrosc. 2007; 15 (2): 147-149.

2. Nicolini AP, Carvalho RT, Ferretti M, Cohen M. Simultaneous bilateral tibial tubercle avulsion fracture in a male teenager: case report and literature review: Case report and literature review. J Pediatr Orthop. 2018; Part B 27 (1): 40-46. doi: 10.1097/BPB.0000000000000313.

3. Hamilton SW, Gibson PH. Simultaneous bilateral avulsion fractures of the tibial tuberosity in adolescence: A case report and review of over 50 years of literature. Knee. 2006; 13 (5): 404-407. doi: 10.1016/j.knee.2006.04.008.
4. Harb Z, Malhi A. Bilateral simultaneous avulsion fractures of the proximal tibia in a 14-year-old athlete with vitamin-D deficiency. Case Rep Orthop. 2015; 783046. doi: 10.1155/2015/783046.

5. Newman C, Musiienko D, Law S. Surgical fixation of bilateral simultaneous avulsion fractures of the proximal tibia in a 12-year-old with history of conservatively managed unilateral tibial avulsion fracture. Case Rep Orthop. 2017; 5925421. doi: 10.1155/2017/5925421.

6. Ergün M, Taskiran E, Ozgürbüz C. Simultaneous bilateral tibial tubercle avulsion fracture in a basketball player. Knee Surg Sports Traumatol Arthrosc. 2003; 11 (3): 163-166. doi: 10.1007/s00167-003-0342-2.

7. Inoue G, Kuboyama K, Shido T. Avulsion fractures of the proximal tibial epiphysis. Br J Sports Med. 1991; 25 (1): 52-56. doi: 10.1136/bjsm.25.1.52.

8. E Albuquerque RP, Giordano V, Carvalho ACP, Puell T, E Albuquerque MIP, do Amaral NP. Simultaneous bilateral avulsion fracture of the tibial tuberosity in a teenager: Case report and therapy used. Rev Bras Ortop. 2012; 47 (3): 381383. doi: 10.1016/s2255-4971(15)30116-6.

9. Narayana Gowda BS, Mohan Kumar J. Simultaneous bilateral tibial tubercle avulsion fracture in a case of pre-existing Osgood-Schlatter disease (OSD). J Orthop Case Rep. 2012; 2 (1): 24-27.

10. Siebert $\mathrm{CH}$, Hofler HR, Hansis M. Bilateral apophyseal rupture of the tibial tuberosity in the athlete. Sportverletz Sportschaden. 1995; 9 (2): 58-61. doi: 10.1055/s-2007-993423.

11. Slobogean GP, Mulpuri K, Alvarez CM, Reilly CW. Comminuted simultaneous bilateral tibial tubercle avulsion fractures: a case report. J Orthop Surg (Hong Kong). 2006; 14 (3): 319-321. doi: 10.1177/230949900601400316.

12. Tulic G, Sopta J, Bumbasirevic M, Todorovic A, Vucetic C. Simultaneous bilateral avulsion fracture of the tibial tubercle in adolescent: a case report. J Pediatr Orthop. 2010; Part B 19 (1): 118-121. doi: 10.1097/BPB.0b013e328331046a.

13. Hanley C, Roche SJ, Chhabra J. Acute simultaneous bilateral avulsion fractures of the tibial tubercles in a 15-year-old male hurler: case report and literature review. Ir J Med Sci. 2011; 180 (2): 589-592. doi: 10.1007/s11845-008-0263-7.

14. Neugebauer A, Muensterer OJ, Buehligen U, Till H. Bilateral avulsion fractures of the tibial tuberosity: a double case for open reduction and fixation. Eur J Trauma Emerg Surg. 2008; 34 (1): 83-87. doi: 10.1007/s00068-007-6154-1.

15. Khoriati AA, Guo S, Thakrar R, Deol RS, Shah KY. Bilateral atraumatic tibial tubercle avulsion fractures: case report and review of the literature. Injury. 2015; 46 (4): 767-769. doi: 10.1016/j.injury.2015.01.012.

16. Borch-Madsen P. On symmetrical bilateral fracture of the tuberositas tibiae and eminentia intercondyloidea. Acta Orthop Scand. 1954; 24 (1): 44-49. doi: 10.3109/17453675408988551.

17. Roy SP, Nag K. Simultaneous bilateral tibial tuberosity avulsion fractures in adolescence: case report and review of 60 years of literature. Injury. 2013; 44 (12): 1953-1955. doi: 10.1016/j. injury.2013.04.006.

18. Nikiforidis PA, Babis GC, Triantafillopoulos IK, Themistocleous GS, Nikolopoulos K. Avulsion fractures of the tibial tuberosity in adolescent athletes treated by internal fixation and tension band wiring. Knee Surg Sports Traumatol Arthrosc. 2004; 12 (4): 271-276. doi: 10.1007/s00167-003-0417-0.

19. Ogden JA, Hempton RJ, Southwick WO. Development of the tibial tuberosity. Anat Rec. 1975; 182 (4): 431-445. doi: 10.1002/ar.1091820404. 
20. Nakase J, Goshima K, Numata H, Oshima T, Takata Y, Tsuchiya $\mathrm{H}$. Precise risk factors for Osgood-Schlatter disease. Arch Orthop Trauma Surg. 2015; 135 (9): 1277-1281. doi: 10.1007/s00402-015-2270-2.

21. Ladenhauf HN, Seitlinger G, Green DW. Osgood-Schlatter disease: a 2020 update of a common knee condition in children. Curr Opin Pediatr. 2020; 32 (1): 107-112. doi: 10.1097/ MOP. 0000000000000842

22. LaMont LE, Doyle SM. Orthopedic aspects of collagen disorders. Curr Opin Pediatr. 2014; 26 (1): 79-84. doi: 10.1097/ MOP.0000000000000035.

23. Adib N, Davies K, Grahame R, Woo P, Murray KJ. Joint hypermobility syndrome in childhood. A not so benign multisystem disorder? Rheumatology (Oxford). 2005; 44 (6): 744-750.

24. Czamara A, Kuzniecow M, Krolikowska A. Arthrofibrosis of the knee joint - the current state of knowledge. Literature review. Ortop Traumatol Rehabil. 2019; 21 (2): 95-106. doi: 10.5604/01.3001.0013.1545.

\section{Conflicto de intereses}

Los autores declaran no tener conflicto de intereses respecto a este trabajo. 